\title{
Combining Coded Signals with Arbitrary Modulations in Orthogonal Relay Channels
}

\author{
Brice Djeumou, ${ }^{1}$ Samson Lasaulce, ${ }^{1}$ and Antoine O. Berthet ${ }^{2}$ \\ ${ }^{1}$ Laboratoire des Signaux et Systèmes, CNRS, Supélec, Universitaire Paris Sud, 91191 Gif-sur-Yvette, France \\ ${ }^{2}$ Départment Télécoms, Supélec, 91191 Gif-sur-Yvette, France \\ Correspondence should be addressed to Brice Djeumou, djeumou@lss.supelec.fr
}

Received 21 March 2008; Accepted 16 July 2008

Recommended by Huseyin Arslan

We consider a relay channel for which the following assumptions are made. (1) The source-destination and relay-destination channels are orthogonal (frequency division relay channel). (2) The relay implements the decode-and-forward protocol. (3) The source and relay implement the same channel encoder, namely, a convolutional encoder. (4) They can use arbitrary and possibly different modulations. In this framework, we derive the best combiner in the sense of the maximum likelihood (ML) at the destination and the branch metrics of the trellis associated with its channel decoder for the ML combiner and also for the maximum ratio combiner (MRC), cooperative-MRC (C-MRC), and the minimum mean-square error (MMSE) combiner.

Copyright (๑) 2008 Brice Djeumou et al. This is an open access article distributed under the Creative Commons Attribution License, which permits unrestricted use, distribution, and reproduction in any medium, provided the original work is properly cited.

\section{Motivations and Technical Background}

We consider orthogonal relay channels for which orthogonality is implemented in frequency [1]. Since the sourcedestination channel is assumed to be orthogonal to the relaydestination channel, the destination receives two distinct signals. In order to maintain the receiver complexity at a low level, the destination is imposed to combine the received signals before applying channel decoding. The relay is assumed to implement the decode-and-forward (DF) protocol. We have at least two motivations for this choice. First, in contrast with the well-known amplify-and-forward (AF) protocol, it can be implemented in a digital relay transceiver. More importantly, whereas the AF protocol imposes the sourcerelay channel to have the same bandwidth as the relaydestination channel, the DF protocol offers some degrees of freedom in this respect. This is a critical point when the cooperative network has to be designed from the association of two existing networks. For instance, if one wants to increase the performance of a digital video broadcasting (DVB) receiver or reach some uncovered indoor areas, a possible solution is to use cell phones, say universal mobile telecommunications system (UMTS) cell phones as relaying nodes. The problem is that DVB signals use a $20 \mathrm{MHz}$ bandwidth (source-relay channel) while UMTS signals have only a bandwidth of $5 \mathrm{MHz}$ (relay-destination channel). The AF protocol cannot be used here; but the DF protocol can be used, for instance, by adapting the modulation of the cooperative signal to the available bandwidth. In this case, the destination has to combine two signals with different modulations.

In this context, one of the issues that needs to be addressed is the design of the combiner. A conventional MRC cannot be used for combining signals with different modulations (except for special cases of modulations). Even if the modulations at the source and relay are identical, the MRC can severely degrade the receiver performance because it does not compensate for the decoding noise introduced by the relay [2-6]. This is why the authors of $[2,4]$ proposed a maximum-likelihood detector (MLD) for combining two BPSK-modulated signals coming from the source and relay. The authors of [6] proposed an improved MRC called C-MRC which aims at maximizing receive diversity. The authors of [3] proposed a linear combiner for which the weights are tuned to minimize the raw bit error rate (BER). The main issue is that one is not always able to explicit the raw BER as a function of the combiner weights whereas the likelihood calculation is more systematic. Additionally, when 
some a priori knowledge is available, the ML metric can be used to calculate an a posteriori probability (APP). In the context of orthogonal $N$-relay channels, the authors of [4] derived two new combiners: the best MRC in the sense of the equivalent signal-to-noise ratio and MMSE combiner. They also assessed the BER performance of the latter and MLD in the uncoded case.

Compared to the afore-mentioned works, this paper also aims at designing a good combiner at the destination but it differs from them on two essential points. (1) The interaction between the combiner and channel decoder is exploited in the sense that we want to express the branch metrics of the trellis associated with channel decoding for the MRC, MMSE combiner, C-MRC, and especially for the ML combiner. (2) When the ML combiner is assumed, the source and relay can use arbitrary modulations (not necessarily BPSK modulations as in $[2,3,5])$ and, more importantly, these can be different.

\section{Signal Model}

At the source, the $L$-information bit sequence $\underline{m}$ is encoded into a sequence of bits $\underline{b}$ and modulated into the transmitted signal $\underline{x}=(x(1), \ldots, x(T))$, where for all $t \in$ $\{1, \ldots, T\}, x(t) \in \mathcal{X}, \mathcal{X}$ is a finite alphabet corresponding to the modulation constellation used by the source and $\mathbb{E}|x(t)|^{2} \leq P_{0}$. At the relay, the message is decoded, reencoded with the same encoder as the source and modulated into the transmitted signal $x_{1}=\left(x_{1}(1), \ldots, x_{1}\left(T_{1}\right)\right)$ where for all $t_{1} \in\left\{1, \ldots, T_{1}\right\}, x_{1}\left(t_{1}\right) \in \mathcal{X}_{1}, \mathcal{X}_{1}$ is a finite alphabet corresponding to the modulation constellation used by the relay, and $\mathbb{E}\left|x_{1}\left(t_{1}\right)\right|^{2} \leq P_{1}$. We denote by $s$ (resp., $r$ ) the number of coded bits conveyed by one source (resp., relay) symbol. By definition: $s=\log _{2}|X|$ and $r=\log _{2}\left|X_{1}\right|$. More specifically, the information bit sequence is assumed to be encoded by a $1 / q$-rate convolutional encoder $\left(q \in \mathbb{N}^{*}\right)$. As the sequence $\underline{x}$ comprises $T$ symbols, we have that $q(k+v)=$ $s T$ where $v$ is the channel encoder memory. Assuming timeselective but frequency-nonselective channels, the baseband signals received by the destination from the source and relay, respectively, write $y_{0}(t)=h_{0} x(t)+z_{0}(t)$ and $y_{1}\left(t_{1}\right)=$ $h_{1} x_{1}\left(t_{1}\right)+z_{1}\left(t_{1}\right)$, where $z_{0}$ and $z_{1}$ are zero-mean circularly symmetric complex Gaussian noises with variances $\sigma_{0}^{2}$ and $\sigma_{1}^{2}$, respectively. The complex coefficients $h_{0}$ and $h_{1}$ represent the gains of the source-destination and source-relay fading channels. For insuring coherent decoding, these two gains are assumed to be known to the receiver and relay, respectively. We define $\gamma_{0}=\mathbb{E}\left|h_{0}\right|^{2}\left(P / \sigma_{0}^{2}\right), \gamma_{1}=\mathbb{E}\left|h_{1}\right|^{2}\left(P / \sigma_{1}^{2}\right), \gamma_{1}^{\prime}=$ $\mathbb{E}\left|h_{1}^{\prime}\right|^{2}\left(P / \sigma_{0}^{2}\right)$, and $\rho_{1}=\mathbb{E}\left|X_{1} X^{*}\right| / P$, where $h_{1}^{\prime}$ is the gain of the source-relay fading channel. Note that, in order to ensure the conservation of the coded bit rate between the input and output of the relay, $s$ and $r$ have to be linked by the following compatibility relation: $s T=r T_{1}$. In the sequel, we will use the quantity $k=1 \mathrm{~cm}(s, r)$, where $\mathrm{lcm}$ is the least common multiple function. For simplicity, we assume that the source and relay use the same channel coder. Therefore, the relay has to use a modulation that is compatible with the source's one. We will also assume that the number of times per second the channel can be used is directly proportional to the available bandwidth. For example, if the source uses a BPSK modulation and the cooperation channel has a bandwidth equal to half the downlink bandwidth, the relay can use a QPSK modulation.

\section{A new Trellis Branch Metric}

\subsection{When the Source and Relay Use Arbitrary and Different Modulations}

In this case, the linear combiners derived by $[3,4,6]$ cannot be used in general. However, provided that the above compatibility condition is met, the ML combiner can be derived as we show now. Let us denote by $\underline{y}_{0}$ and $\underline{y}_{1}$ the sequences of noisy symbols received by the destination from the source and relay, respectively. The discrete optimization problem the ML combiner solves is as follows:

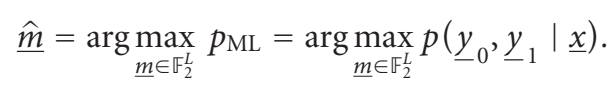

As the reception noises are assumed to be independent, $p_{\mathrm{ML}}=p\left(\underline{y}_{0} \mid \underline{x}\right) p\left(\underline{y}_{1} \mid \underline{x}\right)$. The first term easily writes as

$$
p\left(\underline{y}_{0} \mid \underline{x}\right)=\prod_{t=1}^{T} \frac{1}{\pi \sigma_{0}^{2}} \exp \left(-\frac{\left|y_{0}(t)-h_{0} x(t)\right|^{2}}{\sigma_{0}^{2}}\right) .
$$

In order to express the second term, we introduce a sequence of $T_{1}$ discrete symbols denoted by $\underline{e}_{1}$ which models the residual noise at the relay after the decodingreencoding process. This noise is, therefore, modeled by a multiplicative error term which is not independent of the symbols transmitted by the relay. Additionally, the statistics of this noise are assumed to be known by the destination. For this, one can establish once and for all a lookup table between the source-relay SNR and the bit error rate after reencoding at the relay. The cooperation signal writes then as $y_{1}\left(t_{1}\right)=h_{1} x_{1}\left(t_{1}\right)+z_{1}\left(t_{1}\right)$, where $x_{1}\left(t_{1}\right)=\underline{e}_{1}\left(t_{1}\right) \tilde{x}_{1}\left(t_{1}\right)$ and $\tilde{x}_{1}\left(t_{1}\right)$ is the symbol the relay would generate if there were no decoding error at the relay. For example, when the relay uses a QPSK modulation, $e_{1} \in\left\{1, e^{j(\pi / 2)}, e^{j \pi}, e^{j(3 \pi / 2)}\right\}$. Therefore, we have that $p\left(\underline{y}_{1} \mid \underline{x}\right)=p\left(\underline{y}_{1} \mid \underline{x}_{1}\right)=$ $\sum_{\underline{e}_{1}} p\left(\underline{y}_{1}, \underline{e}_{1} \mid \underline{\tilde{x}}_{1}\right)=\sum_{\underline{e}_{1}} p\left(\underline{e}_{1} \mid \underline{\tilde{x}}_{1}\right) p\left(\underline{y}_{1} \mid \underline{\tilde{x}}_{1}, \underline{e}_{1}\right)$. At this point, we need to make an additional assumption in order to easily derive the path metric of the ML decoder. From now on, we assume that the discrete symbols of the sequence $\underline{e}_{1}$ are conditionally independent. This assumption is very realistic, for example, if the source and relay implement a bit-interleaved coded modulation (BICM) or a trellis-coded modulation (TCM). In the case of the BICM, the channel coder, which generates coded bits, and the modulator are separated by an interleaver. The presence of this interleaver precisely makes the proposed assumption reasonable. Under 
the afore-mentioned assumption, one can expand $p\left(\underline{y}_{1} \mid \underline{x}\right)$ as

$$
\begin{aligned}
p\left(\underline{y}_{1} \mid \underline{x}\right)= & \sum_{\underline{e}_{1}} \prod_{t_{1}=1}^{T_{1}} p\left(e_{1}\left(t_{1}\right) \mid \tilde{x}_{1}\left(t_{1}\right)\right) p\left(y_{1}\left(t_{1}\right) \mid \tilde{x}_{1}\left(t_{1}\right), e_{1}\left(t_{1}\right)\right) \\
= & \sum_{\underline{e}_{1}} \prod_{t_{1}=1}^{T_{1}} p\left(e_{1}\left(t_{1}\right) \mid \tilde{x}_{1}\left(t_{1}\right)\right) \\
& \times \frac{1}{\pi \sigma_{1}^{2}} \exp \left(-\frac{\left|y_{1}\left(t_{1}\right)-h_{1} e_{1}\left(t_{1}\right) \tilde{x}_{1}\left(t_{1}\right)\right|^{2}}{\sigma_{1}^{2}}\right) .
\end{aligned}
$$

The main consequence of this assumption is a significant reduction of the decoder complexity. If the assumption is not valid, the proposed derivation can always be used but the performance gain obtained can be marginal since the errors produced by will not be spread over the data block but rather occurs in a sporadic manner along the block.

In order to express the path metric of a given path in the trellis associated with channel decoding, we need now to link the likelihood expressed above and the likelihood associated with a given bit $b_{j}$, where $j \in\{1, \ldots, k\}$. The reason why we consider subblocks of $k$ bits is that in order to meet the rate compatibility condition, the ML combiner combines the $k_{s}=k / s$ symbols received from the source with the $k_{r}=k / r$ symbols received from the relay. Now for all $(i, j) \in\{0,1\} \times\{1, \ldots, k\}$, let us define the sets $\mathcal{B}_{i}^{(k)}(j)=$ $\left\{\underline{b}^{k}=\left(b_{1}, \ldots, b_{k}\right) \in\{0,1\}^{k}, b_{j}=i\right\}$, a set of subblocks of rs consecutive bits, $X_{i}^{\left(k_{s}\right)}(j)=\left\{\underline{x}^{k_{s}} \in X^{k_{s}}, b_{j}=i\right\}$, and $\mathcal{X}_{1, i}^{\left(k_{r}\right)}(j)=\left\{\underline{\tilde{x}}_{1}^{k_{r}} \in \mathcal{X}_{1}^{k_{r}}, b_{j}=i\right\}$, their equivalents in the source (resp., relay) modulation space. With these notations, the bit likelihood can be expressed as follows:

$$
\begin{aligned}
\lambda\left(b_{j}\right. & =i) \\
& =\ln \left[\sum_{\underline{b}_{1}^{k} \in \mathcal{B}_{i}^{(k)}(j)} p\left(\underline{y}_{0,1}^{k_{s}} \mid \underline{x}_{1}^{k_{s}}\right) p\left(y_{1,1}^{k_{r}} \mid \underline{\underline{x}}_{1,1}^{k_{r}}\right)\right] \\
& =\ln \left[\sum_{\underline{b}_{1}^{k} \in \mathcal{B}_{i}^{(k)}(j)} \prod_{t=1}^{k_{s}} p\left(y_{0}(t) \mid x(t)\right) \prod_{t_{1}=1}^{k_{r}} p\left(y_{1}\left(t_{1}\right) \mid \tilde{x}_{1}\left(t_{1}\right)\right)\right],
\end{aligned}
$$

where we used the notation $\underline{v}_{1}^{n}=(v(1), \ldots, v(n))$. When a BICM is used, the obtained log-likelihood sequence is then de-interleaved and given to a Viterbi decoder.

\subsection{When the Source and Relay Use Arbitrary and Identical Modulations}

The derivation of the coded-bit likelihood in the case where the modulations used by the source and relay are the same is ready since it is special case of derivation conducted previously with $k=s=r$. In this case, both ML and linear combiners can be used since the combination can be performed symbol-by-symbol. The log-likelihood becomes $\lambda\left(b_{j}=i\right)=\ln \left[\sum_{\underline{b}_{1}^{s} \in \mathcal{B}_{i}^{(s)}(j)} p\left(y_{0}(t) \mid x(t)\right) p\left(y_{1}(t) \mid x(t)\right)\right]$, where
TABLE 1: Equivalent channel parameters for the linear combiners.

\begin{tabular}{lcc}
\hline & $h_{\mathrm{eq}}$ & $\sigma_{\mathrm{eq}}^{2}$ \\
\hline MRC & $\frac{a_{0}}{\sigma_{0}^{2}}+\frac{a_{1}}{\sigma_{1}^{2}}$ & $\frac{a_{0}}{\sigma_{0}^{2}}+\frac{a_{1}}{\sigma_{1}^{2}}$ \\
\hline MMSE & $\frac{a_{0}}{\sigma_{0}^{2}}+\frac{a_{1} \rho_{1}^{2}}{\sigma_{1}^{2}+a_{1} P_{0}\left(\alpha_{1}^{2}-\rho_{1}^{2}\right)}$ & $\frac{a_{0}}{\sigma_{0}^{2}}+\frac{a_{1} \rho_{1}^{2}}{\sigma_{1}^{2}+a_{1} P_{0}\left(\alpha_{1}^{2}-\rho_{1}^{2}\right)}$ \\
\hline C-MRC & $a_{0}+\frac{\min \left\{\gamma_{1}^{\prime}, \gamma_{1}\right\}}{\gamma_{1}} a_{1}$ & $a_{0} \sigma_{0}^{2}+\frac{\min \left\{\gamma_{1}^{\prime}, \gamma_{1}\right\}}{\gamma_{1}} a_{1} \sigma_{1}^{2}$
\end{tabular}

$1 \leq j \leq s$. If we further assume that the modulations used are BPSK modulations, the likelihood on the received sequences takes a more explicit form. Indeed, it can be checked that

$$
\begin{aligned}
& \ln \left[p\left(\underline{y}_{0}, \underline{y}_{1} \mid \underline{m}\right)\right] \\
&=-k q \ln (2 \pi)-T \ln \left(\sigma_{0}^{2} \sigma_{1}^{2}\right) \\
&-\sum_{t=1}^{T}\left[\frac{\left|y_{0}(t)-h_{0} x(t)\right|^{2}}{\sigma_{0}^{2}}-\ln \left[\sum_{e=-1,+1} \operatorname{Pr}[\epsilon(t)=e]\right.\right. \\
&\left.\left.\quad \times \exp \left(-\frac{\left|y_{1}(t)-h_{1} \operatorname{ex}(t)\right|^{2}}{\sigma_{1}^{2}}\right)\right]\right],
\end{aligned}
$$

where $\operatorname{Pr}[\epsilon=-1]$ represents the residual bit error rate (after the decoding-reencoding procedure inherent to DF protocol). Denote by $\pi$ the interleaver function such that $t=\pi\left(t_{0}\right)$ and $t_{0}=\pi^{-1}(t)$. Finally, the path metric is merely given by

$$
\begin{array}{r}
\mu^{\mathrm{x}}=\sum_{t_{0}=1}^{T}\left[\frac{\left|y_{0}\left(t_{0}\right)-h_{0} x\left(t_{0}\right)\right|^{2}}{\sigma_{0}^{2}}-\ln \left[\sum_{e=-1,+1} \operatorname{Pr}\left(\epsilon\left(t_{0}\right)=e\right)\right.\right. \\
\left.\left.\times \exp \left(\frac{\left|y_{1}\left(t_{0}\right)-h_{1} \operatorname{ex}\left(t_{0}\right)\right|^{2}}{\sigma_{1}^{2}}\right)\right]\right] .
\end{array}
$$

So the combining and channel decoding are performed jointly by modifying the branch metrics as indicated above.

When using a linear combiner, one has to compute the APP from the equivalent signal at the combiner output. This computation requires the equivalent channel gain and noise. We provide them for each linear combiner considered here. For a given combiner, denote its optimal vector of weights by $\underline{w}=\left(w_{0}, w_{1}\right)$ and rewrite the signal at the combiner output as $y=\sum_{i=0}^{1} w_{i} y_{i}=h_{\mathrm{eq}} x+z_{\mathrm{eq}}$, where $h_{\mathrm{eq}}$ and $z_{\mathrm{eq}} \sim \mathcal{N}\left(0, \sigma_{\mathrm{eq}}^{2}\right)$ are the equivalent channel gain and noise, respectively. The bit $\log$-likelihood can then be easily expressed as $\lambda\left(b_{j}=i\right)=$ $\ln \left[\sum_{x \in X_{i}^{(s)}(j)} p\left(y \mid h_{\mathrm{eq}}, \underline{x}\right)\right]$. Table 1 summarizes the values of these quantities with the notations $a_{0}=\left|h_{0}\right|^{2}$ and $a_{1}=\left|h_{1}\right|^{2}$.

\section{Simulation Example}

For Figures 1 and 2, we assume that the source and the relay implement a 1/2-rate convolutional encoder (4-state encoder 


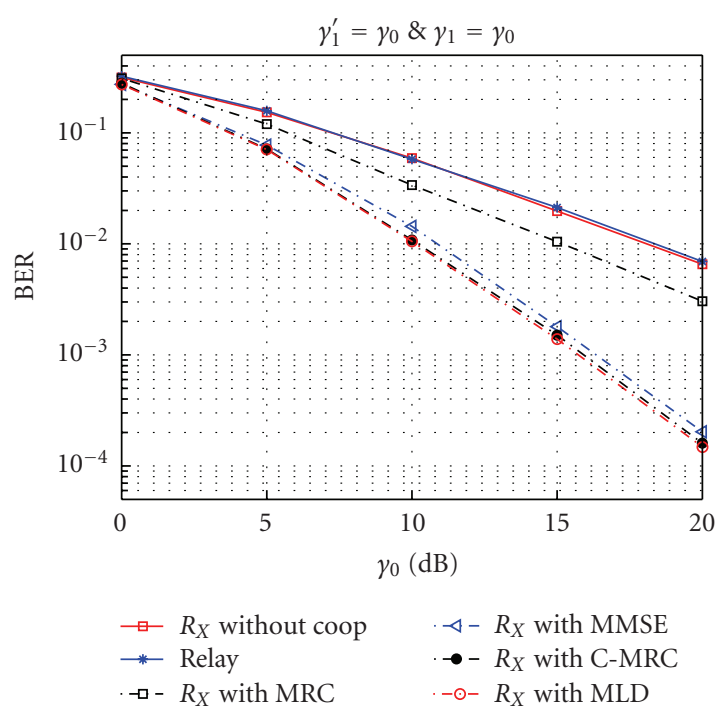

FIGURE 1: BER at the destination with $\gamma_{1}^{\prime}=\gamma_{0}, \gamma_{1}=\gamma_{0}$.

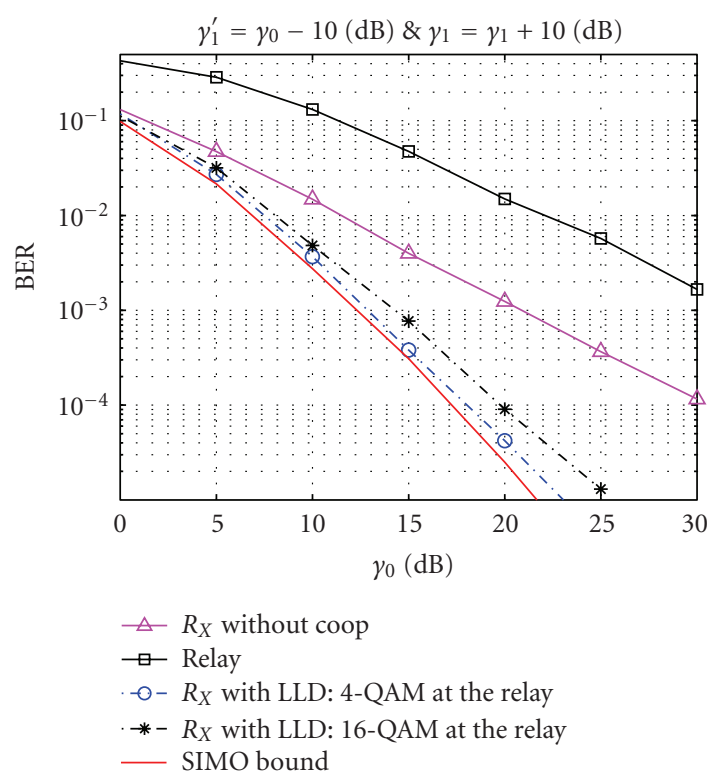

Figure 2: BER at the destination with $\gamma_{1}^{\prime}=\gamma_{0}-10, \gamma_{1}=\gamma_{0}+10$.

with a free distance equal to 5). Frequency nonselective Rayleigh block fading channels are assumed and the data block length is chosen to be 1024. First, we compare the combiners between themselves when both the relay and source use a 4-QAM modulation. Figure 1 represents the BER at the decoder output as a function of $\gamma_{0}=\gamma_{1}$. There are 6 curves: from the top to the bottom, they, respectively, represent the performance with no relay, with the relay associated with the conventional MRC, MMSE, C-MRC, and ML combiners. When implementing the conventional MRC, the receiver does not significantly improve its performance with respect to the noncooperative case whereas the other combiners can provide more than an $8 \mathrm{~dB}$ gain and perform quite similarly. Then (see Figure 2), we evaluate the perfor- mance gain brought by the MLD when the source and relay have to use different modulations: the source implements a BPSK while the relay implements either a 4-QAM or a 16-QAM. The second scenario would correspond to a case where the source-destination channel bandwidth is 4 times larger than the relay-destination channel bandwidth (e.g., $20 \mathrm{MHz}$ versus $5 \mathrm{MHz}$ ). We see that the MLD not only makes cooperation possible but also allows the destination to extract a significant performance gain from it. To have an additional reference, we also represented the performance of the equivalent virtual $1 \times 2 \mathrm{MIMO}$ system, which is obtained for $\gamma_{1}=+\infty$.

\section{Concluding Remarks}

The results provided in this letter and many other simulations performed in the coded case led us to the following conclusion: if the source and relay can use the same modulation, the C-MRC generally offers the best performancecomplexity tradeoff. On the other hand, if the modulations are different, as it would be generally the case when two existing communications systems are associated to cooperate, linear combiners and thus the C-MRC cannot be used in general and the ML combiner is the only implementable combiner.

\section{References}

[1] A. El Gamal, M. Mohseni, and S. Zahedi, "Bounds on capacity and minimum energy-per-bit for AWGN relay channels," IEEE Transactions on Information Theory, vol. 52, no. 4, pp. 15451561, 2006.

[2] J. N. Laneman and G. W. Wornell, "Energy-efficient antenna sharing and relaying for wireless networks," in Proceedings of the IEEE Wireless Communications and Networking Conference (WCNC '00), vol. 1, pp. 7-12, Chicago, Ill, USA, September 2000.

[3] A. Sendonaris, E. Erkip, and B. Aazhang, "User cooperation diversity_part II: implementation aspects and performance analysis," IEEE Transactions on Communications, vol. 51, no. 11, pp. 1939-1948, 2003.

[4] B. Djeumou, S. Lasaulce, and A. G. Klein, "Combining decoded-and-forwarded signals in Gaussian cooperative channels," in Proceedings of the 6th IEEE International Symposium on Signal Processing and Information Technology (ISSPIT '06), pp. 622-627, Vancouver, Canada, August 2006.

[5] D. Chen and J. N. Laneman, "Modulation and demodulation for cooperative diversity in wireless systems," IEEE Transactions on Wireless Communications, vol. 5, no. 7, pp. 1785-1794, 2006.

[6] T. Wang, A. Cano, G. B. Giannakis, and J. N. Laneman, "High-performance cooperative demodulation with decodeand-forward relays," IEEE Transactions on Communications, vol. 55, no. 7, pp. 1427-1438, 2007. 

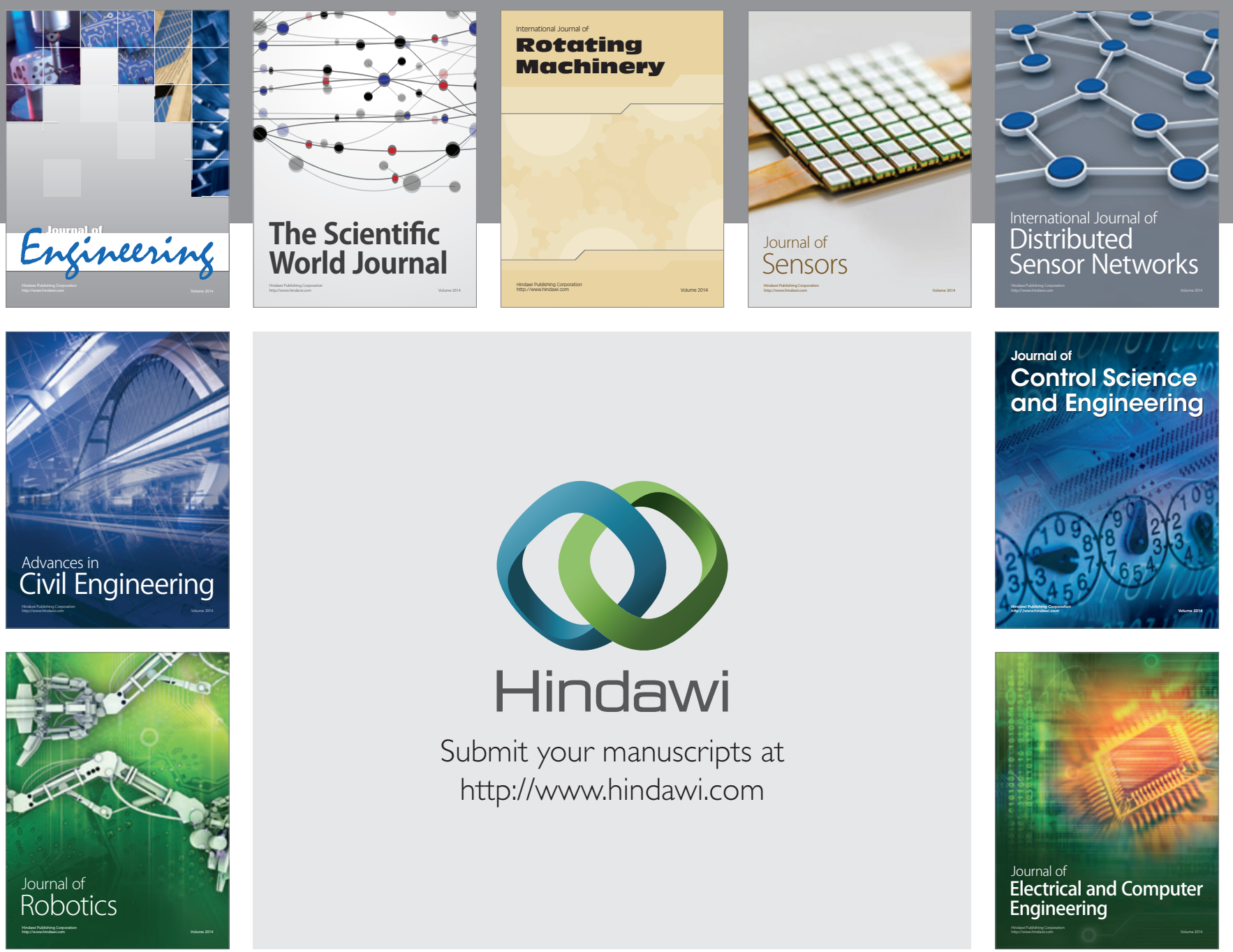

Submit your manuscripts at

http://www.hindawi.com
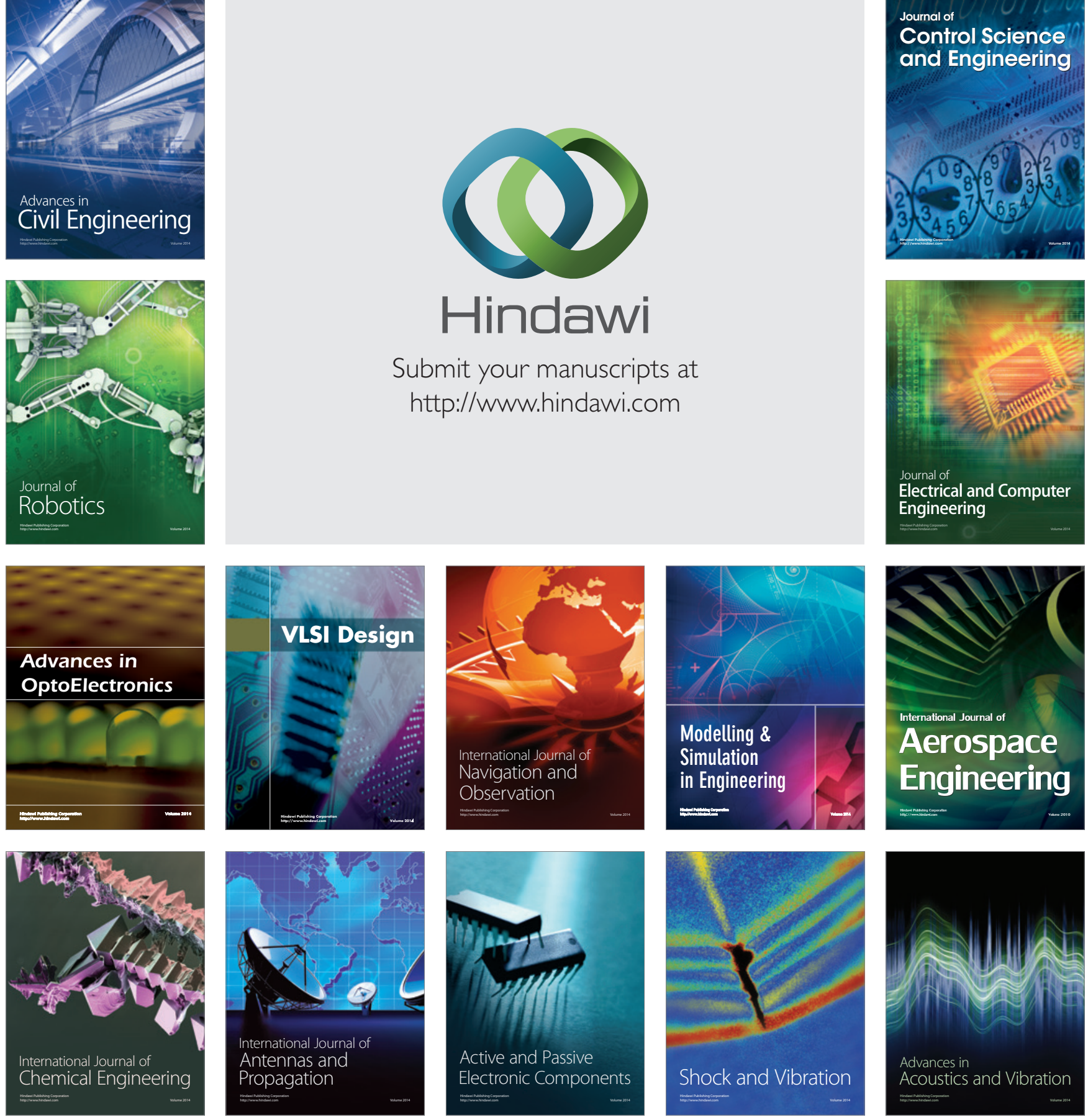